\title{
A two-step method for fast and reliable EUV mask metrology
}

\author{
Patrick Helfenstein*a ${ }^{*}$ Iacopo Mochi ${ }^{a}$, Rajendran Rajeev ${ }^{a}$, Shusuke Yoshitake ${ }^{\mathrm{b}}$, Yasin Ekinci ${ }^{\mathrm{a}}$ \\ ${ }^{\mathrm{a}}$ Paul Scherrer Institute, 5232 Villigen PSI, Switzerland

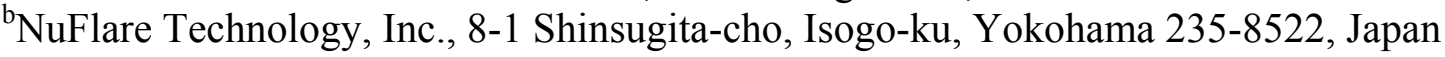 \\ *patrick.helfenstein@psi.ch; https://www.psi.ch/
}

\begin{abstract}
One of the major obstacles towards the implementation of extreme ultraviolet lithography for upcoming technology nodes in semiconductor industry remains the realization of a fast and reliable detection methods patterned mask defects. We are developing a reflective EUV mask-scanning lensless imaging tool (RESCAN), installed at the Swiss Light Source synchrotron at the Paul Scherrer Institut. Our system is based on a two-step defect inspection method. In the first step, a low-resolution defect map is generated by die to die comparison of the diffraction patterns from areas with programmed defects, to those from areas that are known to be defect-free on our test sample. In a later stage, a die to database comparison will be implemented in which the measured diffraction patterns will be compared to those calculated directly from the mask layout. This Scattering Scanning Contrast Microscopy technique operates purely in the Fourier domain without the need to obtain the aerial image and, given a sufficient signal to noise ratio, defects are found in a fast and reliable way, albeit with a location accuracy limited by the spot size of the incident illumination. Having thus identified rough locations for the defects, a fine scan is carried out in the vicinity of these locations. Since our source delivers coherent illumination, we can use an iterative phase-retrieval method to reconstruct the aerial image of the scanned area with - in principle - diffraction-limited resolution without the need of an objective lens. Here, we will focus on the aerial image reconstruction technique and give a few examples to illustrate the capability of the method.
\end{abstract}

Keywords: mask metrology, actinic inspection, EUV, CDI, lensless imaging

\section{INTRODUCTION}

Extreme ultraviolet (EUV) lithography has been developed over the past decades and is considered the most promising technique for upcoming technology nodes with the industry getting ready for high-volume manufacturing. ${ }^{1}$ One of the major challenges to be solved remains the reliable and high-throughput detection of mask defects. ${ }^{2,3}$ Actinic mask metrology, being regarded as an essential part of the EUV mask infrastructure, is a major challenge and methods beyond the state-of-the-art are needed for patterned mask inspection. The established metrology tools such as electron microscopy or DUV scanning microscopy have the disadvantage of being non-actinic, i. e. their imaging response to the absorber, multilayer, and pellicle differs from that of the scanner due to the use of electrons and photons at wavelengths different from the EUV wavelength $(13.5 \mathrm{~nm}) .{ }^{4}$ Phase defects (resulting from bumps and pits within or below the multilayer) are particularly problematic as these are elusive to detect and difficult to repair. ${ }^{5}$

Our reflective EUV mask-scanning lensless imaging tool (RESCAN) aims to solve this issue by using a combination of Scanning Scattering Contrast Microscopy (SSCM) ${ }^{6}$ and Scanning Coherent Diffraction Imaging (SCDI) ${ }^{7}$ methods to provide high-throughput EUV patterned mask defect inspection. SSCM scans the mask using coherent illumination. In our prototype setup, a known defect-free area of the test sample is scanned first and the gathered diffraction patterns are then compared to those of an area that includes defects. This die to die comparison is very fast, since no computation other than summation over the diffraction pattern pixels is necessary and - given a sufficient signal-to-noise ratio - can be used to create a low-resolution defect map with a defect localization accuracy comparable to the illumination spot size. Details about SSCM can be found in a recently published paper. ${ }^{8}$ This rough defect map is used as input for SCDI which will be the main focus of this paper. CDI methods have been invented a long time ago but were long considered impractical for experimental use due to the stringent restrictions of early algorithms. ${ }^{9}$ Fortunately, recent advances in 
phase retrieval algorithms ${ }^{10-13}$ have made SCDI a simple, versatile, and effective technique in various applications ranging from hard X-rays to visible light and electrons. It is also used for EUV mask imaging by many groups ${ }^{14-16}$ and to overcome the resolution limitations of lens-based actinic microscopes. ${ }^{17}$ SCDI uses scattering data recorded with a pixel detector obtained through scanning a finite illumination (probe) across the sample (object) to be imaged. Each subsequent position is chosen so that it overlaps with the previous position to a certain extent. This redundancy in the data is used to retrieve the missing phase and thereby enables robust reconstruction of the complex aerial image of the object without the need for a reference beam or structure. This technique makes it possible to simultaneously obtain both the phase and amplitude information of the sample with a 2D scan whereas lens-based imaging methods need throughfocus scans in order to extract the phase information. Moreover, it has a large depth-of-focus, since the spot size is relatively insensitive to small changes in the large focal distance of the condenser. Since it is a lensless method, it does not need expensive or difficult optics. The resolution of SCDI is typically limited by the NA of the detector, which defines the maximum spatial frequency information acquired. We note that the resolution can also be limited by various other parameters such as the source stability, illumination parameters, scanning method, mechanical instabilities, detector noise, detector pixel number, etc.

Since SCDI offers the aforementioned advantages, it can be a powerful method for actinic EUV mask metrology. The necessary throughput is reached by prepending an SSCM step to limit the area that must be reconstructed. With the current setup of RESCAN, we have been able to reach an on-mask resolution of below $50 \mathrm{~nm}$ using the extended SCDI algorithm detailed below, that encompasses mitigation approaches for instabilities in the sample position and incident illumination.

\section{EXPERIMENTAL SETUP}

The RESCAN experimental chamber is installed at the SIM beamline of the Swiss Light Source that preselects the used photon energy of $92 \mathrm{eV}$ by adjusting the gap of a pure permanent magnet helical undulator with 32 periods of $56 \mathrm{~mm}$ length. The beam is then further filtered using a spherical grating monochromator to provide a coherent beam of EUV light $(\lambda=13.5 \mathrm{~nm})$ with a bandwidth $\lambda / \Delta \lambda \approx 2000$. The beam can be further monochromatized by adjusting the slit openings, albeit with a loss of intensity (cf. Fig. 1 (a)). In Fig. 1 (b), the schematics of the experimental chamber are shown. The chamber is placed $3300 \mathrm{~mm}$ downstream of the beamline intermediate focus position. To increase spatial coherence and clean the beam, a $100 \mu \mathrm{m}$ pinhole was placed at the intermediate focus position and the beam passes through a 5-mm diameter $\mathrm{Si}_{3} \mathrm{~N}_{4}$ membrane for vacuum separation before entering the chamber from the left. The beam is then focused and reflected on the two Mo/Si multilayer mirrors M1 and M2. M1 has a spherical surface with a radius of curvature equal to $220 \mathrm{~mm}$, focusing the beam close to the sample plane after reflection from M2 which has a flat surface and was cut into a crescent moon shape to allow the free passage of the diffracted beam from the sample. The sample was mounted on a piezo-electric $x-y$ stage with a range of $100 \mu \mathrm{m}$ in each direction. The reflected beam from the sample was captured by a $2048 \times 2048$ pixel CCD camera (Princeton Instruments, PI-MTE2048B) cooled to $-40^{\circ} \mathrm{C}$. To maximize the detector's dynamic range, each position was imaged three times with exposure times of 65,500 and 10,000 ms, respectively. Prior to reconstruction, the corresponding background was subtracted from each image and the resulting image normalized to an exposure time of 1 second. Using a simple erosion and dilation filter, the images were then combined into one final diffraction pattern. The diffraction patterns are collected in sequence by moving the sample, actuating the shutter (triggered by the CCD controller) and reading out the CCD data in an automatic manner using EPICS software. The throughput bottleneck is the frame rate of the detector which is below $1 \mathrm{~Hz}$. To this end, PSI is developing a hybrid detector based on a $\mathrm{Si}$ sensor module that delivers acquisition rates of up to $2 \mathrm{kHz}$ and has a dynamic range of $>10^{6} \mathrm{ADC}$ counts, due to an automatically adjustable per-pixel gain. More details about the detector development can be found in refs. 18 and 19.

The test sample used for the data presented here (cf.

Figure 2) was fabricated in-house by spin-coating hydrogen silsesquioxane (HSQ) resist with a thickness of $\sim 60 \mathrm{~nm}$ onto a Si wafer coated with a Si/Mo multilayer. The HSQ absorber structures were then patterned by electron beam lithography and consist of several gratings with programmed defects, a Siemens star, L-shapes, square patterns and several more complicated structures. The four gratings shown have a half-pitch (hp) of $1 \mu \mathrm{m}, 500 \mathrm{~nm}, 250 \mathrm{~nm}, 100 \mathrm{~nm}$ from bottom to the top and left to right, respectively. It should be noted that the sample is not an industrial grade EUV mask and exhibits poor contrast due to the high EUV-transparency of the HSQ resist. 


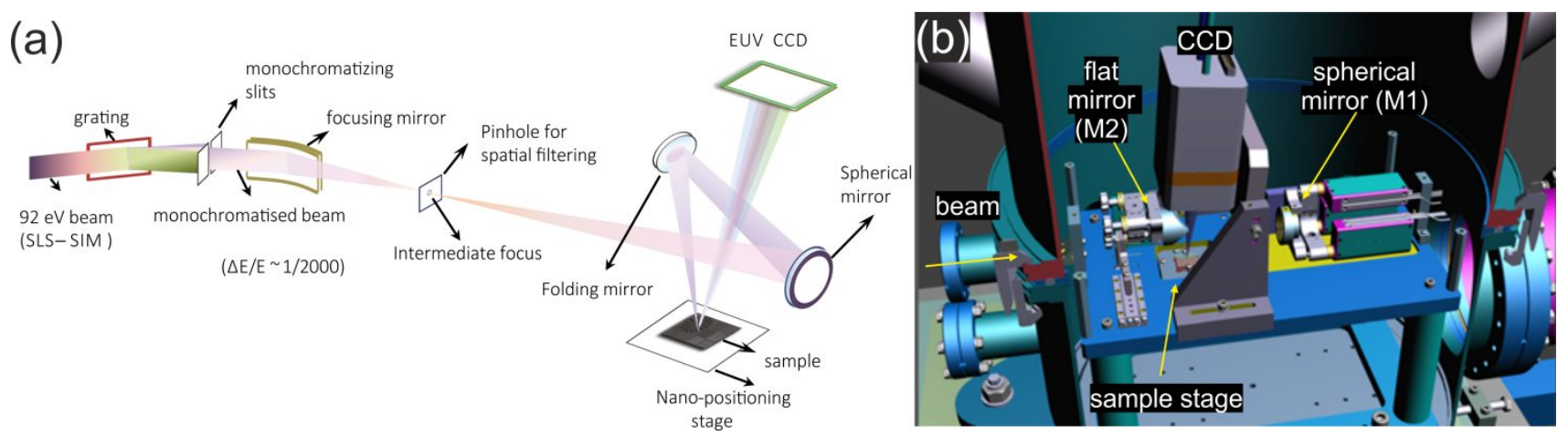

Figure 1 Schematic view of the experimental setup. (a) Schematic setup of the SIM beamline and end-station. The energy of $92 \mathrm{eV}$ is preselected by adjusting the undulator gap and further refined via a spherical grating monochromator (b) End-station optical setup. The beam is focused by a spherical multilayer mirror (M1) and then reflected onto the sample by a flat mirror (M2) at an angle of $6^{\circ}$. After reflection, the resulting diffraction pattern is imaged on the CCD.

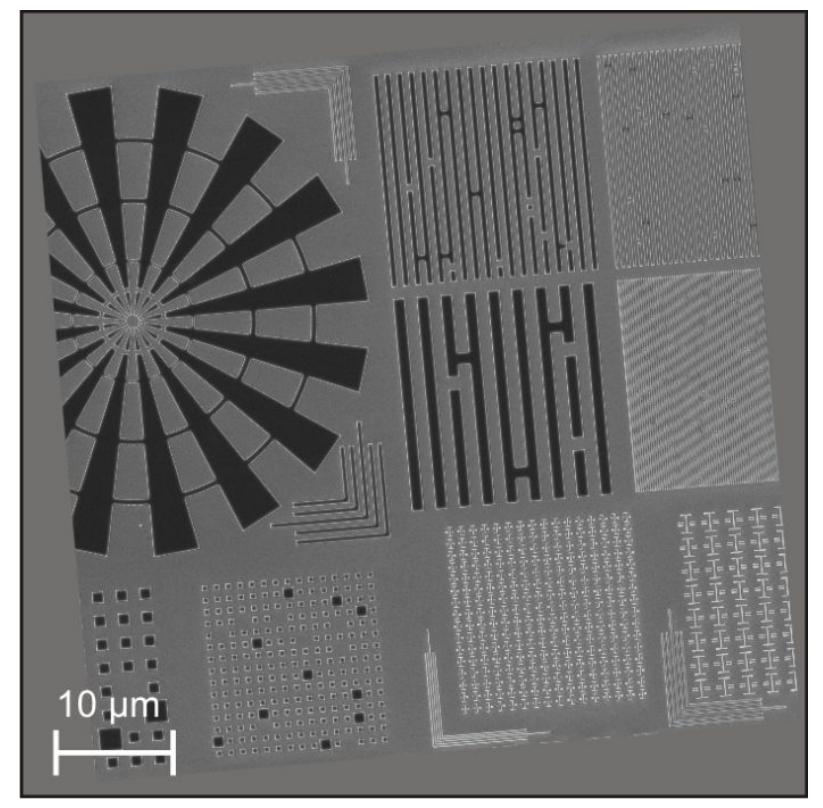

Figure 2. SEM micrograph of the test sample. The HSQ absorber structures were spin-coated on a multilayer wafer and then patterned using electron beam lithography.

\section{RESULTS}

We imaged a $90 \times 90 \mu \mathrm{m}$ area of our HSQ sample mask using a spiral with a step size of $2 \mu \mathrm{m}$. We chose a spiral rather than a raster pattern to avoid the so-called raster grid pathology wherein the scan-points lie on a periodic grid which introduces an additional degree of freedom into the reconstruction and leads to artifacts. ${ }^{12}$ Using the described scan pattern, a total of 4827 diffraction patterns were recorded - taking into account that each of the 1609 positions was 
captured with 3 different exposure times as described above. The total acquisition time was approximately 8 hours, limited mainly by the CCD repetition rate.

Unlike our previous experiments ${ }^{6,7}$, where we had to reduce the incident bandwidth using a combination of Fresnel zone plate and a pinhole mounted close to the sample surface, in this experiment we used a spherical grating monochromator to arrive at a narrow energy spectrum. To further clean the beam, a $100 \mu \mathrm{m}$ diameter circular pinhole was inserted at the intermediate focus position of the beamline (cf. Fig. 1 (a)) approximately $3300 \mathrm{~mm}$ upstream of the sample position. Due to the monochromatic beam, the reconstruction quality is improved compared to previous results where strong artifacts were visible in the background between the structures. Figure 3 illustrates the progress of the RESCAN imaging results over the course of one year. RESCAN 1 refers to the setup at the XIL-II interference lithography beamline, where no monochromator was available, but we managed to reduce the bandwidth from an initial $4 \%$ (leftmost image) to $0.5 \%$ focusing the incident beam on a $4 \mu \mathrm{m}$ pinhole close to the sample plane via a Fresnel zone plate (second image from the left). RESCAN 2 refers to the current end station installed at the SIM beamline. The progress from left to right is due to small changes in the beamline setup, such as the addition of the above mentioned $100 \mu \mathrm{m}$ pinhole at the intermediate focus and advances in the iterative reconstruction algorithms.
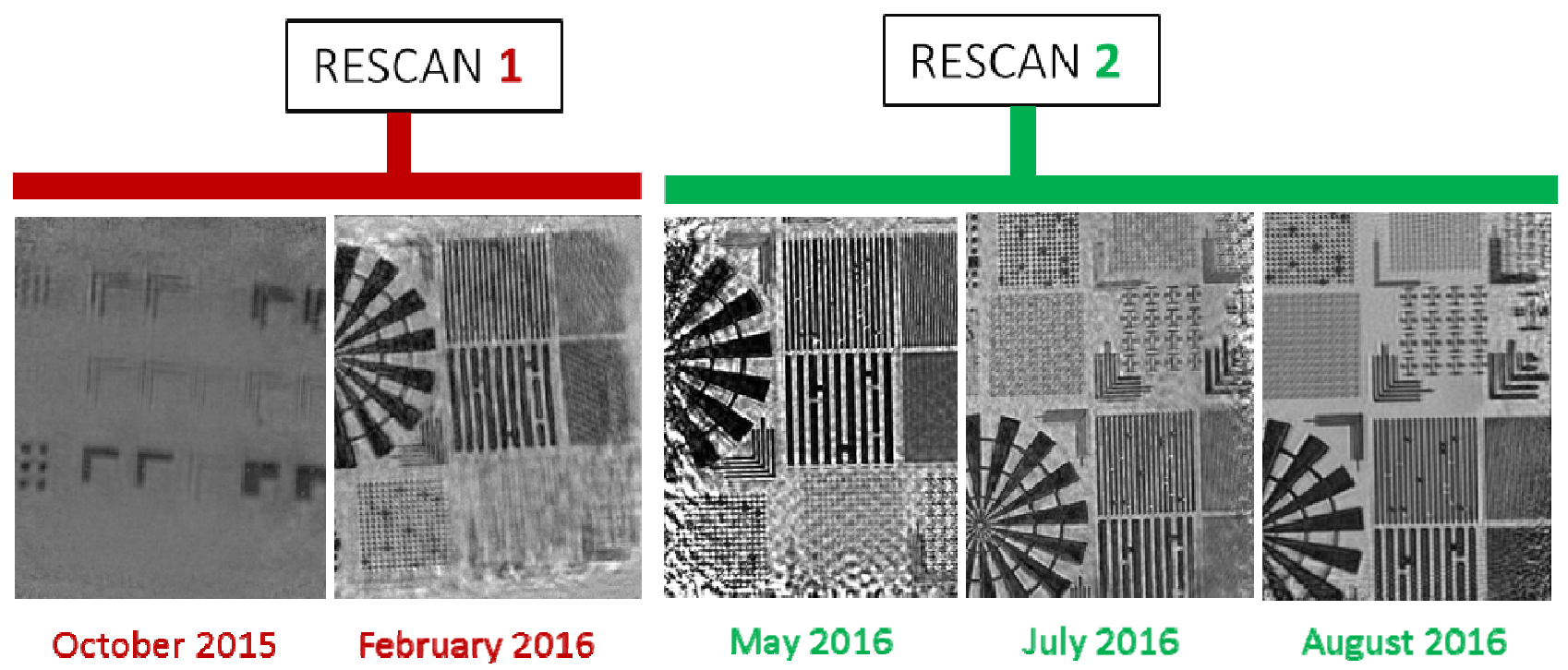

Figure 3 Timeline of the RESCAN imaging results. RESCAN 1 refers to the initial end station installed at the XIL-II interference lithography beamline, where no monochromator was available. A Fresnel zone plate was used to focus the beam on a pinhole close to the sample plane. The improvement from the leftmost image to the one on the right is due to the reduction in pinhole size from $10 \mu \mathrm{m}$ to $4 \mu \mathrm{m}$. RESCAN 2 is our current end station setup using a spherical mirror to focus the monochromatized beam on the sample. The improvements from the left to the right-hand side were achieved by employing multiple exposures to extend the dynamic range, inserting a pinhole at the intermediate focus for spatial filtering and advances in the algorithms, respectively.

To carry out the reconstruction of our imagined sample we use a version of the difference map algorithm adapted for SCDI. The quality of the resulting reconstruction depends on various factors, but two main factors that operate on different time scales can be readily identified:

i) partial coherence; short time-scale

ii) probe variations; long time-scale

In this context, the short time-scale describes variations with a frequency above the exposure time whereas the long timescale encompasses changes in the experimental conditions that vary slowly during the time it takes to acquire all positions of a complete scan. Considerable effort has been spent on the mitigation of artifacts in the reconstruction that are due to partial coherence and modeling the system as a state-mixture and then reconstructing the resulting states using 
multiple modes for the incident illumination or the imaged sample has shown promising results. ${ }^{20}$ While this enhancement to SCDI reconstruction allows superior resolution for partially coherent systems, it does not take into account variations of the probe for the duration of the experiment, which can last for multiple hours. To cover the effects of probe variation, we follow a simple approach. Given the mechanical stability of the optics and of the sample stage, it is safe to assume that probe variations are only noticeable over the range of several minutes. We therefore allow the algorithm to keep multiple independent probes in memory. Each is used for its own portion of the recorded diffraction patterns and updated accordingly. This way, the probe instability during the scan duration is taken into account without any increase in computation time.

Even though great care was taken to minimize vibrations in the experiment to improved data quality, a small improvement in resolution of the reconstructed aerial image could still be gained using multiple probe modes (cf. Fig. 4 (b)). On the other hand, the long acquisition time of 8 hours cannot be accurately reproduced by using a constant probe throughout the experiment. Here we permit the algorithm to use 40 independent probes (one separate probe for every $\sim 12$ min slice of the experiment). This strongly reduced artifacts due to the variation in the incident beam and allows for increased resolution as show in Fig. 4 (c). The highest quality reconstruction with increased contrast is achieved by using multiple probes, each with its own modes (cf. Fig. 4 (d)). Measuring the edge profile resulted in an overall resolution $<50 \mathrm{~nm}$. Assuming a diffraction-limited system, the maximum achievable solution with this setup is $38 \mathrm{~nm}$ for a NA of 0.18 defined by the detector size of $25.4 \mathrm{~mm}$ and a distance to the sample of $70 \mathrm{~mm}$.
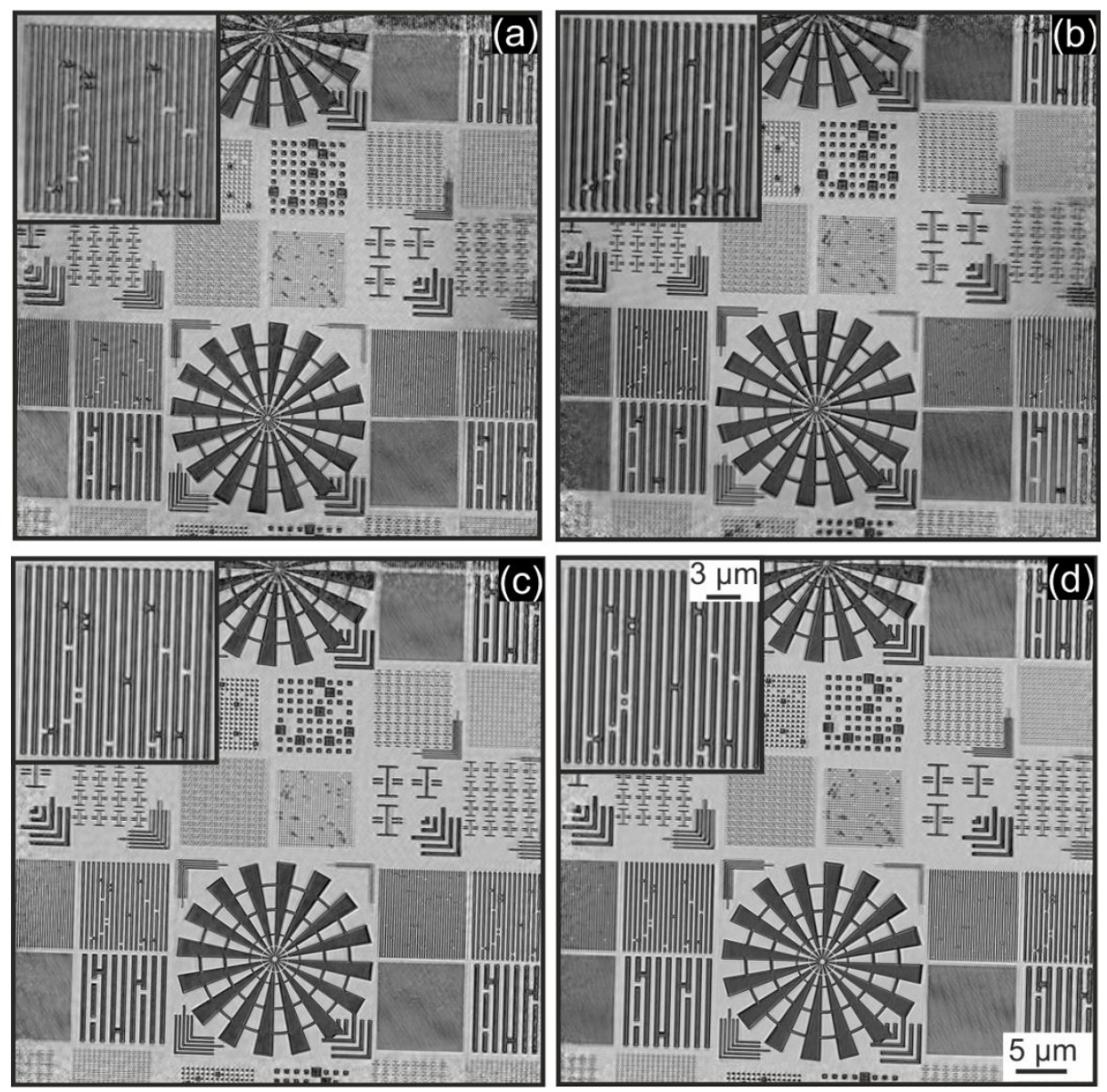

Figure 4 SCDI reconstruction of the same data with different approaches. (a) standard SCDI method, (b) using multiple probe modes, (c) using multiple independent probes (d) using multiple independent probes, each with its own probe modes gives the best resolution and contrast. The inset depicts a close-up of the $500 \mathrm{~nm}$ half pitch grating. 


\section{CONCLUSIONS AND OUTLOOK}

We have shown that - given a rough defect location map - SCDI can be used to reliably identify mask defects. In order to reach the throughput and sensitivity requirements of the semiconductor industry, all parts of the RESCAN chamber will have to be upgraded. Most importantly the sample stage that currently has a limited x,y range of $200 \mu \mathrm{m}$ and the detector. In the next step, the CCD detector will be replaced by a hybrid silicon detector. The new detector will allow a frame-rate of up to $2 \mathrm{kHz}$ and a dynamic range of $10^{6}$ electrons. The higher dynamic range will eliminate the need for multiple exposures necessary when using a CCD. This alone will increase the scan speed by a factor of 12,000 . Also, it is planned to extend the stage range to approximately $1 \mathrm{~cm}$ in both $\mathrm{x}$ and $\mathrm{y}$ direction and add a $\mathrm{z}$-stage to allow for throughfocus scans.

The reconstruction was carried out on a dual-CPU Linux system with a total of 40 cores and 512 GB of RAM. Using a reconstruction size of $400 \mathrm{px}$, it takes less than 3 minutes to complete 300 SCDI iterations, which is usually more than sufficient to reach convergence. The given time is valid for a single mode reconstruction using only one probe and increases by approximately $30 \%$ for each additional probe mode used, but is independent of the number of probes. In a next step, parts of the code will be ported to the GPU which will increase the computation speed significantly, but as it scales roughly reciprocal with the number of available cores, the limiting factors are much more likely to be found in the hardware than the software.

\section{ACKNOWLEDGEMENTS}

The authors would like to express their gratitude to Armin Kleibert and Carlos Vaz who instructed us about the various settings of the SIM beamline and were always available for further questions, Markus Kropf and Jonas Woitkowiak for their invaluable help with instrumentation and numerous suggestions that led to substantial improvements in the collected data, Jose Gabadinho for taking care of connecting all parts of our endstation to EPICS and Pascal Schifferle for helping with the setup of our endstation at the SIM beamline of SLS.

\section{REFERENCES}

[1] Hendrickx, E., Gronheid, R., Hermans, et al., "Readiness of EUV Lithography for Insertion into Manufacturing: The IMEC EUV Program", Journal of Photopolymer Science and Technology 26, 587-593 (2013).

[2] Uzzel, D., Garetto, A., Magnusson, K., Tabbone, G., "A novel method for utilizing AIMS ${ }^{\text {TM }}$ to evaluate mask repair and quantify over-repair or under-repair condition", Proc. SPIE 8880, 888029 (2013).

[3] Goldberg, K. A., Mochi, I., Benk, M., et al., "Commissioning an EUV mask microscope for lithography generations reaching $8 \mathrm{~nm}$ ”, Proc. SPIE 8679, 867919 (2013).

[4] Mochi, I., Goldberg, K. A., La Fontaine, et al., "Actinic imaging of native and programmed defects on a full-field mask", Proc. SPIE 7636, 76361A (2010).

[5] Goldberg, K., Mochi, I., "Wavelength-Specific Reflections: A Decade of EUV Mask Inspection Research", International Workshop on EUV Lithography (2009).

[6] Mohacsi, I., Helfenstein, P., Rajeev, R., and Ekinci, Y., "Scanning Scattering Contrast Microscopy for actinic EUV mask inspection", Proc. SPIE 9778 (2016)

[7] Helfenstein, P., Mohacsi, I., Rajeev, R., Ekinci, Y., "Scanning coherent diffractive imaging methods for actinic extreme ultraviolet mask metrology", J. Micro/Nanolith. MEMS MOEMS 15 (3), 034006 (2016)

[8] Mochi, I., Helfenstein, P., Mohasi, I., Rajeev, R., Shusuke, Y., Ekinci, Y., "RESCAN: An actinic lensless microscope for defect inspection of EUV reticles", Proc. SPIE 10143 (2017)

[9] Hoppe, W., "Beugung im inhomogenen Primärstrahlwellenfeld. I. Prinzip einer Phasenmessung von Elektronenbeungungsinterferenzen". Acta Crystallographica A 25 (4), 495 (1969).

[10] Rodenburg, J. M. and Faulkner, H. M. L., "A phase retrieval algorithm for shifting illumination", Applied Physics Letters 85(20), 4795 (2004).

[11] Thibault, P., Dierolf, M., Menzel, A., Bunk, O., David, C., and Pfeiffer, F., "High-resolution scanning X-ray diffraction microscopy", Science (New York, N.Y.) 321, 379-82 (2008).

[12] Thibault, P., Dierolf, M., Bunk, O., et al., "Probe retrieval in ptychographic coherent diffractive imaging", Ultramicroscopy 109, 338 (2009) 
[13] Maiden, A. M. and Rodenburg, J. M., "An improved ptychographical phase retrieval algorithm for diffractive imaging", Ultramicroscopy 109, 1256 (2009).

[14] Seaberg, M. D., Adams, D. E., Townsend, E. L., et al., "Ultrahigh $22 \mathrm{~nm}$ resolution coherent diffractive imaging using a desktop $13 \mathrm{~nm}$ high harmonic source", Opt. Express 19, 22470-9 (2011).

[15] Gardner, D. F., Zhang, B., Seaberg, M. D., et al., "High numerical aperture reflection mode coherent diffraction microscopy using off-axis apertured illumination", Opt. Express 20, 19050-9 (2012).

[16] Juschkin, L., Loetgering, L., Rudolf, D., et al., "Tabletop coherent diffraction imaging with a discharge plasma EUV source", Proc. SPIE 8849, 88490Y (2013)

[17] Wojdyla, A. J., Benk, M. P., Goldberg, K. A., "Fourier Ptychography imaging for the study of EUV lithography photomasks", Proc. SPIE 9776 (2016)

[18] Redford, S., Bergamaschi, A.., Brückner, M., "Calibration status and plans for the charge integrating JUNGFRAU pixel detector for SwissFEL," J Inst 11(11), C11013-C11013 (2016).

[19] Rajeev, R., Mochi, I., Helfenstein, P., Mohacsi, I., Redford, S., Mozzanica, A., Schmitt, S., Shusuke, Y., Ekinci, Y., "Towards a stand-alone high-throughput EUV actinic photomask inspection tool - RESCAN", Proc. SPIE 10145 (2017)

[20] Thibault, P. and Menzel, A., "Reconstructing state mixtures from diffraction measurements", Nature (494), 68-71 (2013) 